Kardiologe 2021 · 15:462

https://doi.org/10.1007/s12181-021-00498-1

Online publiziert: 30 . Juli 2021

(c) Deutsche Gesellschaft für Kardiologie - Herzund Kreislaufforschung e.V. Published by Springer Medizin Verlag GmbH, ein Teil von Springer Nature - all rights reserved 2021

\section{Erratum zu: Wearable-basierte Detektion von Arrhythmien}

\section{Positionspapier der Deutschen Gesellschaft für Kardiologie}

\section{Erratum zu:}

Kardiologe 2021

https://doi.org/10.1007/s12181-021-

00488-3

In dem ursprünglichen Artikel wurde der Name des Autors Hendrik Schneider falsch geschrieben. Bitte beachten Sie die korrigierte Schreibweise. Der Originalbeitrag wurde korrigiert.

Der vollständige und korrigierte Artikel steht Ihnen auf www.springermedizin.de zur Verfügung. Bitte geben Sie dort den Beitragstitel in die Suche ein.

\section{Korrespondenzadresse}

Univ.-Prof. Dr. med. Christian Veltmann Hannover Herzrhythmus Centrum, Klinik für Kardiologie und Angiologie, Medizinische Hochschule Hannover

Carl-Neuberg-Str. 1, 30625 Hannover, Deutschland

veltmann.christian@mh-hannover.de

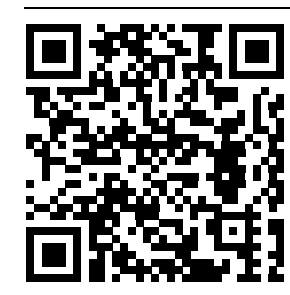

QR-Code scannen \& Beitrag online lesen

Die Online-Version des Originalartikels ist unter https://doi.org/10.1007/s12181-021-00488-3 zu finden. 